\title{
Une bonne surveillance de la grossesse pour préparer l'accouchement
}

Population Council

Follow this and additional works at: https://knowledgecommons.popcouncil.org/departments_sbsr-rh

Part of the International Public Health Commons, Maternal and Child Health Commons, Public Health Education and Promotion Commons, and the Social and Behavioral Sciences Commons How does access to this work benefit you? Let us know!

\section{Recommended Citation}

Population Council. 2010. "Une bonne surveillance de la grossesse pour préparer l'accouchement," brief. Dakar: Population Council. 


\section{Sénégal}

SMNI/PF/PALUDISME

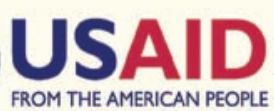

La Situation 2

\section{Une bonne surveillance de la grossesse pour préparer l'accouchement}

\author{
Le suivi prénatal a pour objectif d'aider la femme à mener sa \\ grossesse à terme et à accoucher dans les meilleures conditions \\ de sécurité pour elle et son enfant.
}

\section{Contexte}

Menée en Janvier 2007 dans les sept (7) régions d'intervention du programme Santé de l'USAID dans le cadre de la mise en œuvre des activités préliminaires de la composante Santé Maternelle, Néonatale, Infantile/Planification Familiale/Paludisme (SMNI/PF/PALU), l'Etude d'Analyse Situationnelle (EAS), avait pour but de fournir des informations de base devant aider le programme à identifier des domaines prioritaires d'intervention ou à réajuster les interventions initialement proposées.

Plus spécifiquement, l'EAS avait pour objectif d'aider la Composante à développer le paquet intégré de services SR à mettre en place au niveau des points de prestations de service (PPS) et à élaborer les ressources didactiques nécessaires pour le renforcement des compétences des prestataires et l'organisation des services.

La méthodologie utilisée a été basée sur l'approche classique des EAS qui repose sur : i) l'inventaire des structures pour analyser leur capacité fonctionnelle à offrir les services de SR ; ii) l'observation de la pratique des prestataires pour évaluer leur performance en matière d'offre de service $\mathrm{SR}$; iii) l'interview des prestataires pour évaluer leur connaissance des procédures de services SR et leurs attitudes en perceptions sur les questions de SR; iv) l'interview de sortie des clientes pour analyser la qualité de l'offre de service SR sous la perspective de la cliente.

L'offre de service de Consultation Prénatale (CPN) a été un des volets de ce paquet intégré

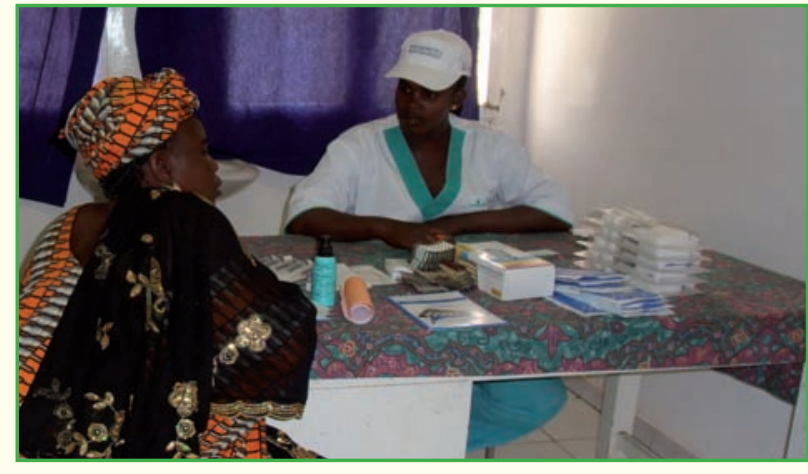

de services qui a fait l'objet de cette étude. Pour apprécier la qualité des services, neuf cent onze (911) consultations prénatales ont été observées et huit cents soixante-trois (863) interviews de sortie de clientes ont été réalisées au niveau de deux cents soixanteneuf (269) PPS.

\section{Résultats}

\section{Disponibilité des services de CPN}

\begin{tabular}{|lll|}
\hline Indicateurs de disponibilitél & & $\%$ \\
fonctionnalité & & \\
PPS offrant les services de CPN & Centre de santé & 100 \\
\cline { 2 - 3 } & Poste de santé & 100 \\
\hline $\begin{array}{l}\text { Prestataires formés sur la } \\
\text { surveillance de la grossesse, de } \\
\text { l'accouchement et du post partum }\end{array}$ & & 94 \\
\hline $\begin{array}{l}\text { Score de disponibilité du matériel } \\
\text { d'examen général }\end{array}$ & Centre de santé & 93 \\
\cline { 2 - 3 } & Poste de santé & 95 \\
\hline $\begin{array}{l}\text { Score de disponibilité du matériel } \\
\text { d'examen gynécologique }\end{array}$ & Centre de santé & 80 \\
\cline { 2 - 3 } & Poste de santé & 73 \\
\hline $\begin{array}{l}\text { Score disponibilité matériel } \\
\text { prévention des infections }\end{array}$ & Centre de santé & 69 \\
\cline { 2 - 3 } & Poste de santé & 63 \\
\hline $\begin{array}{l}\text { PPS disposant de supports IEC sur } \\
\text { la Maternité à Moindre Risque }\end{array}$ & Boîte à image & 23 \\
\cline { 2 - 3 } & Affiches & 42 \\
\cline { 2 - 3 } & Dépliants & 14 \\
\hline
\end{tabular}

Les données du tableau indiquent une effectivité de l'offre de services de CPN au niveau de tous les PPS visités (100\%). Aussi, 
la grande majorité du personnel (94\%) a reçu une formation de base sur la surveillance de la grossesse. On observe par ailleurs une bonne disponibilité du matériel d'examen général et dans une moindre mesure du matériel d'examen gynécologique. Toutefois, la disponibilité du matériel de prévention des infections et des supports IEC sur la maternité à moindre risque devra être renforcée.

\section{Connaissance et pratique des prestataires}

L'analyse des scores révèle des écarts importants par rapport au niveau acceptable de performance fixé à $85 \%$. Le score de connaissance des procédures de la CPN est de $59 \%$ tandis que celui de la pratique est de $39 \%$.

Ces résultats indiquent ainsi des écarts importants entre la connaissance des procédures de CPN et leur application. écarts sont surtout visibles si l'on se réfère aux étapes spécifiques de la procédure de la CPN. En dehors de l'étape de l'accueil, toutes les autres étapes ne sont pas bien suivies par les prestataires durant la consultation.

Les étapes de l'interrogatoire, de la prévention des infections et de l'information des clientes sont les moins bien suivies. Cette situation pourrait s'expliquer d'une part par le fait que peu de

\begin{tabular}{|ll|}
\hline Score moyen de performance (compétence pratique) & $\%$ \\
\hline Interrogatoire & 87 \\
\hline Examen Physique & 32 \\
\hline Prévention des Infections & 51 \\
\hline Test et examens de laboratoire & 30 \\
\hline Prescription & 35 \\
\hline Enregistrement des données sur les supports & 58 \\
\hline Conseils/éducation de la cliente & 66 \\
\hline Score moyen (en \%) & 20 \\
\hline
\end{tabular}

prestataires $(45 \%)$ ont bénéficié d'une orientation sur les Politiques, Normes et Protocoles en matière d'offre de services de SR et, d'autre part, par l’irrégularité de la supervision. Seul 26\% des PPS visités ont reçu une supervision sur la CPN.

\section{Qualité et intégration des services}

L'analyse de la qualité des CPN à travers la perspective des clientes indique une faible interaction entre les clientes et les prestataires durant la consultation. Seul 22\% des clientes ont déclaré que le prestataire les a informé sur le déroulement de l'examen et seulement $\quad 10 \%$ d'entre elles ont indiqué que le prestataire leur a demandé si elles avaient d'éventuelles questions.

L'intégration des services demeure également faible. La prise en charge des autres besoins de SR des clientes demeure faible, notamment la prévention du paludisme chez la femme enceinte, la préparation de l'accouchement et la PF. Seul $37 \%$ des clientes ont reçu le TPI sous TDO tandis que seul 14\% d'entre elles ont reçu des conseils sur la préparation de l'accouchement et $10 \%$ sur la PF.

\section{Recommandations}

- Disséminer les nouveaux PNP au niveau de tous les PPS et orienter les prestataires sur les nouveaux PNP en vue d'améliorer l'application des procédures

- Assurer la supervision et le suivi post formation des prestataires

- Orienter les prestataires sur l'approche d'identification systématique des besoins de la cliente pour renforcer l'intégration des services. reflètent pas nécessairement les vues de l'USAID ou du Gouvernement des Etats-Unis. 